\section{SF005/\#192 STRATEGY FOR MIS UTERINE MANIPULATION IN CERVICAL CARCINOSARCOMA}

${ }^{1} \mathrm{M}$ Simonsen*, ${ }^{2} \mathrm{~B}$ Migliavacca, ${ }^{1} \mathrm{~A}$ Teixeira, ${ }^{2} \mathrm{P}$ Doria, ${ }^{2} \mathrm{~F}$ Asanuma, ${ }^{2} \mathrm{~A}$ Santiago, ${ }^{1} \mathrm{~A}$ Ferreira, ${ }^{1} \mathrm{M}$ Lanoni, ${ }^{3} \mathrm{I}$ De andrade, ${ }^{3} \mathrm{~L}$ Silva, ${ }^{3} \mathrm{~B}$ Fernandes, ${ }^{3} \mathrm{M}$ De Mello Amaral, ${ }^{1} \mathrm{M}$ Samora. ${ }^{1}$ Hospital 9 de Julho, Gynecologic Oncology, São Paulo, Brazil; ${ }^{2}$ Hospital 9 de Julho, Gynecology, São Paulo, Brazil; ${ }^{3}$ IAMSPE, Gynecology, São Paulo, Brazil

\subsection{6/ijgc-2021-IGCS.49}

Introduction Uterine manipulation could be associated with worst outcomes even in endometrial cancer, as suggested recently. In this video we demonstrated simple techniques to overcome the potential tumor spillage during uterine manipulation.

Description LAGOH 57 yo had a low differentiated adenocarcinoma involving uterine isthmus with $5,1 \times 6,4 \mathrm{~cm}$ with endometrial, cervical and right parametrial involvement. Pathologic report suggested an endometrial origin of the tumor. We proposed to do a radical hysterectomy and retroperitoneal lymph node dissection Our team offered to the patient minimally invasive surgical approach and regarding the big dimension of the tumor's cervical component we opted to avoid tumor spillage during uterine manipulation with the following measures: we used a big polypropylene graduated cylinder and fixed the device in the 4 cervical quadrants with stiches. For mobilizing uterine corpus, we used an hysterometer involved with bladder catheter placed in the uterus cavity. Radical hysterectomy, para-aortic lymph node dissection and pelvic lymph node debulking were performed uneventfully in $5 \mathrm{~h} 10 \mathrm{~min}$. Pathologic report accused carcinosarcoma with pelvic lymph nodes involvement, parametrial infiltration and free margins (FIGO IIIC). After 6 cycles of carboplatin/paclitaxel and adjuvant radiotherapy, LAGOH maintains one year of outpatient follow-up without evidence of recurrence.

Conclusion While data related with the impact of uterine manipulation in endometrial/uterine corpus cancer is still not available in multiple prospective trials, it is rational to develop strategies to optimize safety of the procedure.

\section{SF006/\#205 UPFRONT SURGICAL MANAGEMENT OF LARGE OR NECROTIC MALIGNANT MIXED MULLERIAN TUMORS OF THE ENDOMETRIUM BY A MINIMALLY INVASIVE APPROACH}

${ }^{1} \mathrm{G}$ Feuer*, ${ }^{2} \mathrm{M}$ Mousavi, ${ }^{2} \mathrm{~N}$ Lakhi. 'Atlanta Gynecologic Oncology, Northside Hospital, Atlanta, USA; ${ }^{2}$ New York Medical College, Department of Obstetrics and Gynecology, Staten Island, USA

\subsection{6/ijgc-2021-IGCS.50}

Introduction Malignant Mixed Mullerian Tumors (MMMT) often present with very large, necrotic tumor burden in the uterus that leads to dilation and effacement of the cervix. In patients with this presentation, conventional hysterectomy poses a much greater challenge as the ureters are comprised by the mass of the tumor and are at an increased risk for injury. Given this surgical challenge, many of these patients may begin with neoadjuvant chemoradiation. However, these treatment modalities are associated with significant toxicity and negatively impact patient quality of life. Therefore, we present a minimally invasive robotic surgical approach that aims to optimize quality of life without sacrificing prognosis. Description The patient had biopsy proven MMMT. Pre-operative CT scan showed an enlarged uterus with no evidence of extrauterine disease. On presentation, the cervix was dilated and effaced with extruding disease. Disease was initially reduced transvaginally. Robotic approach included 1) upfront vascular control of pedicles, 2) radical hysterectomy with complete dissection of the ureters secondary to the dilated and effaced cervix, 3) infracollic omentectomy, 4) lymph node dissection, and 5) appendectomy.

Conclusion The patient was debulked to zero residual disease. She was discharged the following day ad had no post-operative complications. A robotic approach for staging and complete cytoreduction of bulky Malignant Mixed Mullerian Tumors is feasible with good postoperative outcome.

\section{SF007/\#206 A SYSTEMATIC APPROACH FOR ACHIEVING OPTIMAL CYTOREDUCTION OF LOCALLY ADVANCED ENDOMETRIAL CANCER BY A ROBOTIC APPROACH}

${ }^{1} \mathrm{G}$ Feuer*, ${ }^{2}$ Mousavi, ${ }^{2} \mathrm{~N}$ Lakhi. 'Atlanta Gynecologic Oncology, Northside Hospital, Atlanta, USA; ${ }^{2}$ New York Medical College, Department of Obstetrics and Gynecology, Staten Island, USA

\subsection{6/ijgc-2021-IGCS.51}

Introduction This video demonstrates surgical techniques that can be utilized to optimally cytoreduced locally advanced endometrial cancer via a robotic approach.

Description The patient had biopsy proven endometrial adenocarcinoma. Pre-operative CT scan indicated omental caking with involvement of the pelvic peritoneum and uterosacral ligaments. The patient had diagnostic laparoscopy which showed disease limited to the pelvis. Robotic surgical techniques demonstrated include 1) upfront vascular control of pedicles, 2) lateral mobilization of ureters for management of uterosacral disease, 3) peritonectomy for excision of peritoneal lesions, and 4) infracollic omentectomy.

Conclusions/Implication The patient was debulked to zero residual disease. She was discharged the following day ad had no post-operative complications. A robotic approach for staging and complete cytoreduction of locally advanced endometrial cancer is feasible with good postoperative and oncological outcome. This vidoe presents a step by step aproach for handeling these challenging cases.

\section{SF008/\#514 EXTRAPERITONEAL SENTINEL LYMPH NODE BIOPSY BY VAGINAL NATURAL ORIFICE TRANSLUMINAL ENDOSCOPIC SURGERY (VNOTES) IN PATIENTS WITH NON-PROLAPSED UTERUS AND LOW-RISK ENDOMETRIAL CANCER}

${ }^{1} \mathrm{YY}$ Ju*, ${ }^{1} \mathrm{SJ}$ Park, ${ }^{1} \mathrm{HS}$ Kim, ${ }^{1} \mathrm{~J}-\mathrm{W}$ Kim, ${ }^{2} \mathrm{GW}$ Yim. ' Seoul National University Hospital, Department of Obstetrics and Gynecology, Seoul, Korea, Republic of; ${ }^{2}$ Dongguk University Ilasan Hospital, Department of Obstetrics and Gynecology, Goyang, Korea, Republic of

\subsection{6/ijgc-2021-IGCS.52}

Introduction To evaluated the feasibility of extraperitoneal sentinel lymph node biopsy (SLNB) by vaginal natural orifice transluminal endoscopic surgery (vNOTES) in patients with a non-prolapsed uterus and low-risk endometrial cancer.

Methods A 54-year old woman visited because of vaginal bleeding that started three months ago. Endometrial biopsy revealed grade 1 endometrioid endometrial cancer, and imaging studies showed no abnormal finding. We tried vNOTES 
staging operation with extraperitoneal SLNB. First, each $1 \mathrm{ml}$ of indocyanine green (ICG) diluted at $1.25 \mathrm{mg} / \mathrm{ml}$ was injected into the submucosa and stroma at 3 and 9 o'clock of the cervix. Second, we opened the mid-vagina between the cervix and vaginal orifice where the pelvic floor muscles were palpated. Third, a space was secured by placing a finger upward and outward after ensuring the opening space of the mid-vagina. Thereafter, we inserted single port trocar through the opening, and then we found the para-cervix including the pelvic autonomic nerves, and the obturator nerve. After peeling off the pelvic vessels and nerves and ureter, we found the sentinel lymph node near the external iliac vessel, and resected it. Then, we performed vNOTES hysterectomy, and she was discharged on the second day of surgery without any complications.

Conclusions vNOTES staging operation with extraperitoneal SLNB may be feasible, and the determination over the extraperitoneal opening may be important to ensure the adequate view to identify the pelvic structure for patients with a nonprolapsed uterus and low-risk endometrial cancer.

\section{SF009/\#460 MINIMALLY INVASIVE SECONDARY CYTOREDUCTIVE SURGERY FOR CELIAC AND CARDIOPHRENIC ISOLATED NODAL RECURRENCE OF OVARIAN CANCER}

${ }^{1} V$ Gallotta, ${ }^{2} \mathrm{M}$ Loverro*, ${ }^{3} \mathrm{R}$ Ergasti, ${ }^{4} \mathrm{D}$ Nachira, ${ }^{1} \mathrm{C}$ Conte, ${ }^{2} \mathrm{~V}$ Gallitelli, ${ }^{5} \mathrm{G}$ Scaglione, ${ }^{3} \mathrm{~A}$ Fagotti, ${ }^{2} \mathrm{G}$ Scambia. ${ }^{1}$ Fondazione Policlinico Universitario Agostino Gemelli, IRCCS, Gynecologic Oncology, Rome, Italy; ${ }^{2}$ Università Cattolica del Sacro Cuore, Department of Woman and Child Health and Public Health, Woman Health Area, Fondazione Policlinico Universitario A. Gemelli Irccs, Roma, Italy; ${ }^{3}$ Fondazione Policlinico Universitario A. Gemelli, IRCCS, Department of Woman and Child Health and Public Health, Rome, Italy; ${ }^{4}$ Fondazione Policlinico Universitario A. Gemelli IRCCS, Roma, Italy; Università Cattolica del Sacro Cuore Sede di Roma, Roma, Italy, Department of Thoracic Surgery, Rome, Italy; ${ }^{5}$ Fondazione Policlinico Universitario A. Gemelli IRCCS, Roma, Italy, Gynecopathology and Breast Pathology Unit, Roma, Italy

\subsection{6/ijgc-2021-IGCS.53}

Introduction Despite the advances in cytoreductive efforts and frontline chemotherapy in advanced ovarian cancer (OC), recurrence is a common event, with $>70 \%$ of women experiencing relapse within two years of from diagnosis. The standard treatment for recurrent ovarian cancer (ROC) patients has been traditionally represented by systemic chemotherapy; however, this concept has been recently recognized as presenting a greater level of complexity given the influence of histotype, status of BRCA genes, previous antiangiogenetic treatment and pattern of relapse presentation. Several retrospective studies, as well as randomized prospective trials suggested that secondary cytoreductive surgery (SCS) could provide better oncological outcomes in platinum-sensitive ROC patients, in case of complete cytoreduction, which has to be considered the goal to be achieved.

Description As far as lymph node relapse is concerned, some biological and clinical lines of evidence suggest that lymph node recurrences from OC would be better managed with SCS than medical treatment alone, given a relatively more indolent clinical behaviour compared to parenchymal and peritoneal disease. However, the documentation of lymph nodes metastasis in the hepatoceliac and cardiophrenic region at the time of SCS might be considered as a challenging clinical and surgical scenario. Although surgical management by minimally invasive surgery (MIS) could be expected to represent a demanding task in SCS, this video provides a step-by-step description of the surgical technique adopted for hepatoceliac and cardiophrenic lymph nodes resection.

Conclusion MIS is feasible and could be a viable option for selected cases of ROC, minimizing the intra- and post- operative complications.

\section{SF010/\#77 LAPAROSCOPIC SENTINEL LYMPH NODE BIOPSY FOR STAGE I ENDOMETRIAL CANCER: DUAL TRACER METHOD}

M Slimane, M Ghalleb*, H Bouaziz, M Bouhani, R Chargui, K Rahal. Salah Azaiez Institute of Oncology, Surgical Oncology, Tunis, Tunisia

\subsection{6/ijgc-2021-IGCS.54}

Introduction Sentinel lymph node (SLN) biopsy is a new trending option in the management of stage I endometrial cancer. The first results are showing non inferiority when compared to the classic pelvic lymphadenectomy dissection and also a decrease of morbidity especially lymphedema. Our Aim is to Share one of our cases with the IGCS community.

Description We report the case of a 50 year old women with a stage Ia Endometrial cancer. She was included into our institution Trial to see the feasibility of SLN biopsy in endometrial cancer. The patients have been treated laparoscopically and the Dual tracer method was used to detect the SLN. A four trocars approach was used. A $10 \mathrm{~mm}$ trocars in the umbilicus and one in the left iliac fossa, Two five $\mathrm{mm}$ in the hypogastric region and right iliac fossa. After careful abdominal exploration we detected the blue dye in the left iliac region. We started with opening the left pelvic wall peritoneum. Careful dissection of a blue enlarged lymph node. After the extraction the blue lymph node was also found to have a high radioactivity. The frozen section was negative. As a part of our protocol the women had a full lymph-node dissection and no metastatic lymph-node was found.

Conclusion SLN biopsy in stage one endometrial cancer seems to be technically feasible. We are waiting for the result of our trial to implemented in our current standards of care.

\section{SF011/\#197 TRANSVAGINAL ENDOSCOPIC RESECTION (TVER) OF INVASIVE VAGINAL LESION}

${ }^{1} \mathrm{~N}$ Dogra*, ${ }^{2} \mathrm{R}$ Linder, ${ }^{2} \mathrm{~J}$ Murphy, ${ }^{2} \mathrm{~T}$ Zigras. ${ }^{1}$ University of Toronto, Obstetrics and Gynecology, Toronto, Canada; ${ }^{2}$ Trillium Health Partners, Credit Valley Hospital, Gynecology Oncology, Mississauga, Canada

\subsection{6/ijgc-2021-IGCS.55}

Objective This video demonstrates a novel surgical technique for excision of an invasive vaginal lesion using a single port laparoscopic access device.

Study Methods A 31 year old female with a history of SLE on azathioprine presented with recurrent HSIL of cervix. A LEEP showed microinvasion measuring $2 \mathrm{~mm}$ and a vaginal fornix biopsy showed HSIL, but was clinically suspicious for invasion. The patient underwent a transvaginal endoscopic resection (TVER) of the lesion for diagnostic purposes. A single port laparoscopic access device was used for colpopneumo-occlusion. The endoscope was inserted into the vagina, and the $1.5 \mathrm{~cm}$ lesion was resected with margins of 5 